\title{
Formation of a gold-carbon dot nanocomposite with superior catalytic ability for the reduction of aromatic nitro groups in water $\uparrow$
}

Received 31st March 2014 Accepted 20th May 2014

DOI: $10.1039 / c 4 r a 02837 f$

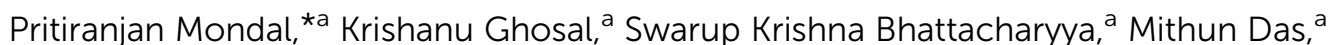
Abhijit Bera, ${ }^{a}$ Debabrata Ganguly, ${ }^{a}$ Pawan Kumar, ${ }^{d}$ Jaya Dwivedi, ${ }^{d}$ R. K. Gupta, ${ }^{\text {b }}$ Angel A. Marti, ${ }^{c}$ Bipin Kumar Gupta ${ }^{\star d}$ and Subhabrata Maiti ${ }^{a}$

www.rsc.org/advances

We report the synthesis of a gold-carbon dot nanocomposite and its utility as a recyclable catalyst for the reduction of aromatic nitro groups. The presence of carbon dots on gold nanosurfaces enhanced the reduction rate by two-fold.

Nanomaterials have emerged as new prospects across scientific disciplines owing to their specific physicochemical and optoelectronic properties. In this context, since the pioneering work of Haruta, researchers have found the usefulness of nanomaterials for catalyzing chemical reactions. ${ }^{1}$ At present, noble metallic nanoparticles like gold and silver are being used in catalyzing diversified class of reactions ranging from carbon monoxide oxidation, $\mathrm{C}-\mathrm{C}$ coupling to hydrogenation. ${ }^{1-3}$ The advantages of using gold nanostructures in modulating catalysis lie in their straightforward synthesis in different forms, stability, and surface functionalization, among others. ${ }^{4,5}$ Typically, smaller nanoparticles show higher catalytic ability in various kinds of reactions. ${ }^{5}$ However, in the case of redox reactions, anisotropic nanostructures with high electron transporting ability such as nanocages or nanoboxes have been observed to enhance the catalytic rate. ${ }^{4}$

Amalgamation of carbon nanostructure and metal nanoparticle is providing some exceptional features in terms of their catalytic, electronic or photovoltaic properties. ${ }^{6,7}$ To this end, among the family of nanodimensional allotropes of carbon, carbon dots (CDs) (which are new but exciting) are showing interesting potential in applications ranging from sensing,

\footnotetext{
${ }^{a}$ Department of Applied Chemistry and Industrial Chemistry, Ramakrishna Mission Vidyamandira, Belurmath, Howrah-711 202, India. E-mail: pritiranjanmondal21@ gmail.com;dganguly93@gmail.com

${ }^{b}$ Department of Chemistry, Pittsburg State University, Pittsburg, KS 66762, USA

${ }^{c}$ Dept. of Chemistry and Bioengineering, Rice University, Houston, TX 77005, USA

${ }^{d}$ National Physical Laboratory (CSIR), Dr K S Krishnan Road, New Delhi 110012, India.E-mail: bipinbhu@yahoo.com

$\dagger$ Electronic supplementary information (ESI) available: Details of synthesis of GNPs and CDs, other experimental details, TEM images, UV-vis spectra, kinetics graph, see DOI: $10.1039 / \mathrm{c} 4 \mathrm{ra02837f}$
}

biomedical devices to photocatalytic applications owing to their intrinsic photophysical properties. ${ }^{8}$ CDs can also exhibit high electron transporting ability as they have an electron rich graphite core. At this point, a nanocomposite comprising CDs and an anisotropic gold nanospecies (like GNR) is of high interest for catalyzing redox type reactions. GNR stabilized with cationic cetyltrimethylammonium bromide (CTAB) can be conjugated with carbon dots having anionic carboxylate functional groups. In principle, the designed composite might offer several advantages: (i) the presence of an anisotropic gold nanospecies (aGNS), which is already known for its high catalytic efficiency due to the presence of different facets; (ii) the availability of the graphite core of CDs, which can expedite the transfer rate of electrons; and most importantly, (iii) the hydrophobic domain between aGNS and CDs can help trap hydrophobic molecules, which would allow aqueous catalytic reactions of water-insoluble compounds. Thus, an aGNS-CD nanocomposite is an interesting approach for the development of a superior nanocatalyst. Therefore, a gold-CD nanocomposite is an interesting approach for the development of a superior nanocatalyst.

Initially, we synthesized aGNS with a transverse surface plasmon peak ( $\left.\lambda_{\text {trans }}, \mathrm{SPR}\right)$ at $520 \mathrm{~nm}$ and a longitudinal peak ( $\lambda_{\text {long}}$ ) at $695 \mathrm{~nm}$ using standard protocols (Fig. 1a). ${ }^{10}$ The details of the synthetic procedure can be found in the ESI. $\dagger$ Transmission electron microscopy (TEM) images revealed that the width of the aGNS (among some other gold nanospecies like nanocubes) is $10 \pm 2 \mathrm{~nm}$ with an aspect ratio of $2.8 \pm 0.3$ (Fig. $1 \mathrm{~b}$ and $\mathrm{c}$ and S1a, ESI $\dagger$ ). The synthesized aGNS are highly stable as they are stabilized by the quaternary ammonium group of cetyltrimethylammonium bromide (CTAB) ${ }^{\mathbf{1 0}}$

In order to produce the aGNS-CD nanocomposite, we designed carbon dots functionalized with a long chain anionic carboxylate (Fig. 1d). CDs were synthesized by thermal coupling between sodium-salt of 11-aminoundecanoic acid and citric acid as discussed elsewhere (Fig. 1d). ${ }^{11}$ Here citric acid acts as the source of the carbon core and 11-aminoundecanoic acid is covalently linked with the carbon nanoparticle via amide bonds. 


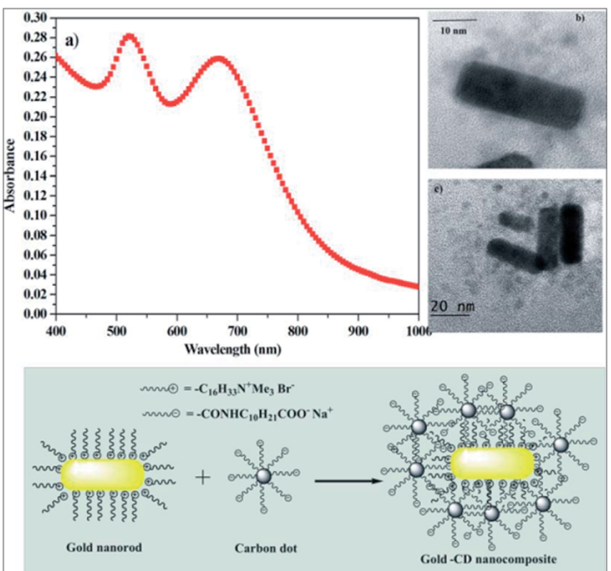

Fig. 1 (a) UV-vis spectra, (b and c) TEM images of synthesized aGNS$C D$ nanocomposite $\left([\mathrm{Au}]=100 \mu \mathrm{M},[\mathrm{CD}]=100 \mu \mathrm{g} \mathrm{mL}^{-1}\right.$ ) and (d) schematic representation of the formation of the aGNS-CD nanocomposite.

The size of the CDs was $3 \pm 1 \mathrm{~nm}$ as found from TEM images (Fig. S1b, ESI $\dagger$ ). Moreover, a negative zeta potential $(\zeta)$ value of $-23.8 \pm 2 \mathrm{mV}$ confirmed the presence of a carboxylate moiety on the surface of the CDs.

The synthesized aGNS solution was centrifuged at 14000 rpm for $90 \mathrm{~min}$ to remove excess CTAB, and the residual material was redispersed in aqueous phosphate buffer $(\mathrm{pH}$ $7.0,25 \mathrm{mM}$ ). This buffered aGNS solution was then used to make the conjugate with CDs functionalized with a carboxylate moiety. The nanoconjugate was prepared by mixing aGNS and CD solutions in phosphate buffer at different ratios. The formation of the aGNS-CD nanoconjugates was confirmed both by spectroscopic and microscopic methods. The strong photoluminescence properties of the CDs (emission and excitation wavelength maxima $=420$ and $340 \mathrm{~nm}$ ) were quenched in the presence of aGNS, as gold nanomaterials are known to quench the emission of fluorophores in close proximity (Fig. 2). ${ }^{5}$ The blue-shift in emission maxima (by almost $20 \mathrm{~nm}$, from $420 \mathrm{~nm}$ to $400 \mathrm{~nm}$ ) in the presence of aGNS is likely due to the interaction between nanogold and CDs. In addition, the TEM images (Fig. 1b and c) show the occurrence of CDs over the gold surface, which is consistent with the formation of the gold-CD composite. We propose that the electrostatic attractive force between cationic aGNS and anionic CDs, as well as the hydrophobic attraction of the long carbon chain, play crucial roles in nanocomposite formation (Fig. 1d).

To test the catalytic ability of the synthesized nanocomposite, we chose the reduction of $p$-nitrophenol as the model reaction. ${ }^{12}$ The conversion of $p$-aminophenol from its nitro analogue is a reaction of versatile applications that includes antipyretic or analgesic drug development, dye industry, and corrosion removal. ${ }^{13,14}$ Moreover, the reaction does not proceed in the absence of any catalyst. The progression of the reaction can be easily monitored by observing the rate at which the characteristic $400 \mathrm{~nm}$ peak of $p$-nitrophenol decreases as it converts to $p$-aminophenol with the generation

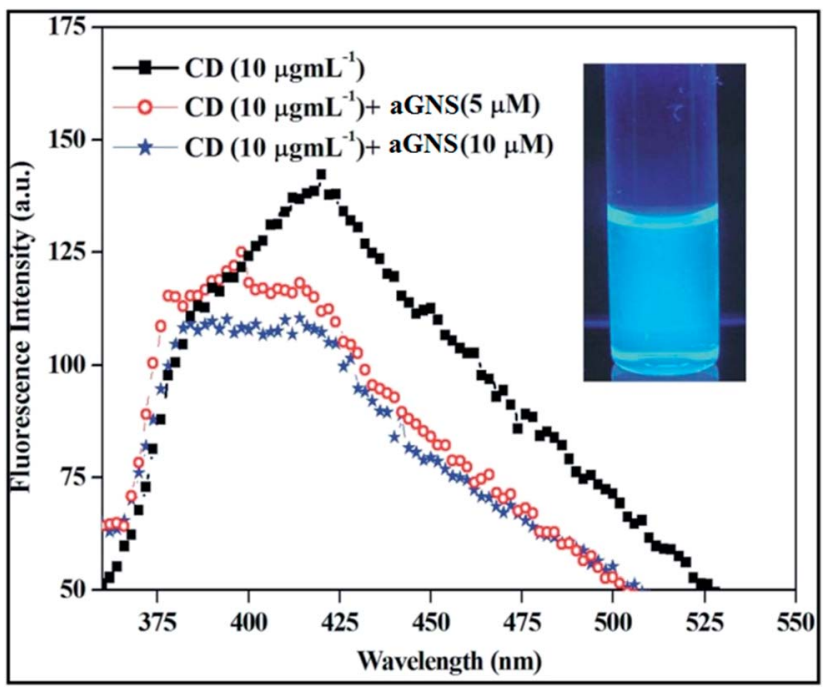

Fig. 2 Fluorescence spectra of CD in absence and presence of aGNS of varying concentration (Inset: Visual fluorescent image of CD).

of a new peak at $300 \mathrm{~nm}$ (Fig. S2 and S3a, ESI $\dagger$ ). The reaction followed pseudo first order kinetics as the concentration of $\mathrm{NaBH}_{4}$ is in excess (almost 1000 times higher) of that of $p$ nitrophenol.

Initially, we monitored the apparent rate constant $\left(k_{\mathrm{app}}\right)$ of the reaction in the presence of varying concentrations of aGNS $([\mathrm{Au}]=10-100 \mu \mathrm{M})$. We have observed an increase in $k_{\text {app }}$ (from 0.05 to $0.072 \mathrm{~s}^{-1}$ ) with increasing Au concentration up to $50 \mu \mathrm{M}$, and then it remained almost constant (Table S1, ESI $\dagger$ ). At this point, the gold-CD composite was fabricated by varying $\mathrm{CD}$ concentration from 12.5 to $100 \mu \mathrm{g} \mathrm{mL}{ }^{-1}$, while the concentration of aGNS was kept constant $(50 \mu \mathrm{M})$. Increasing the aGNS concentration resulted in colloidal instability of the composite. Interestingly, an increase in the $\mathrm{CD}$ concentration in the goldCD composite (up to $50 \mu \mathrm{g} \mathrm{mL} \mathrm{m}^{-1}$ ) led to a 2 -fold increase in the $k_{\text {app }}$ from 0.072 to $0.143 \mathrm{~s}^{-1}$, while a further increase in the CD concentration $\left(100 \mu \mathrm{g} \mathrm{mL} \mathrm{m}^{-1}\right)$ had almost no effect on the $k_{\text {app }}$ at $0.135 \mathrm{~s}^{-1}$ (Fig. 3 and S3, ESI $\dagger$ ). However, the presence of only CDs led to an almost negligible effect towards catalyzing the reaction (Fig. S3, ESI $\dagger$ ).

Furthermore, we also monitored the recyclability of the gold-CD nanocomposite in catalyzing the reduction. Our experiments showed that the catalytic ability remained at about $80 \%$ even after completing the fourth reduction cycle (Fig. 4). In fact, the TEM image of the gold-CD nanocomposite revealed the encirclement of the CDs on the gold nanosurface even after the fourth cycle (Fig. S4, ESI $\dagger$ ). However, the gradual loss in the catalytic ability might be due to detachment of parts of CDs (probably during purification of the composite by centrifugation after each run) from the gold nanosurface and the corresponding decrease in electron transfer efficiency by the CDs as the number of cycle increases. This cyclic stability and retainment of the catalytic activity of the nanocomposite is of high significance in terms of its use as a potential catalyst. 


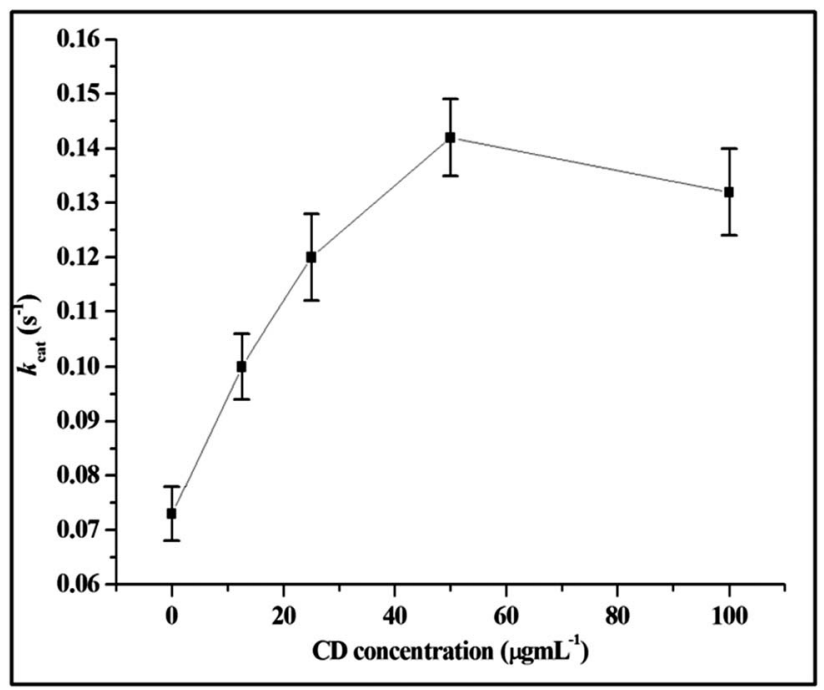

Fig. 3 Variation in $k_{\text {app }}$ of $p$-nitrophenol reduction by $\mathrm{NaBH}_{4}$ in the presence of aGNS-CD nanocomposite in aqueous phosphate buffer $(\mathrm{pH} 7,25 \mathrm{mM})$ at $25^{\circ} \mathrm{C}([\mathrm{Au}]=50 \mu \mathrm{M}$ in all aGNS-CD composites).

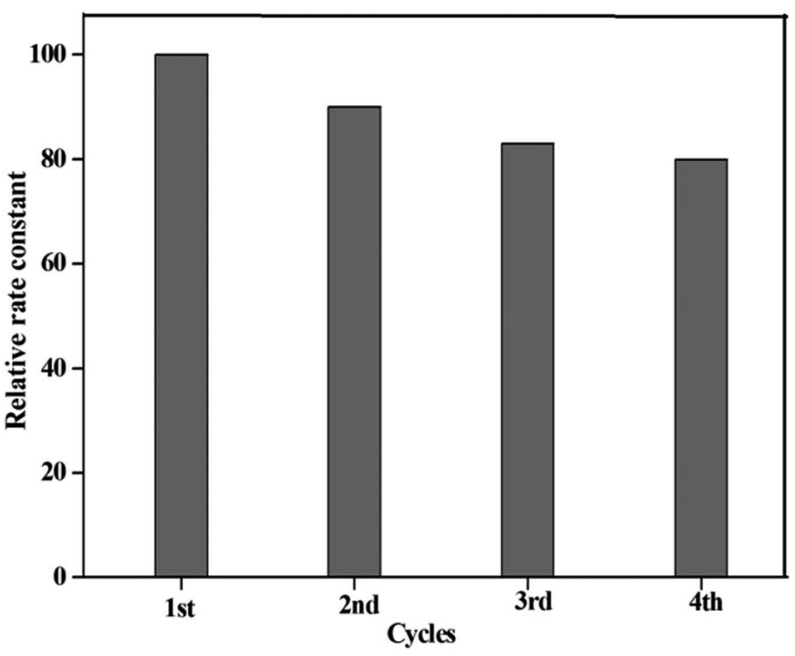

Fig. 4 Recyclability of the gold-CD nanocomposite as a catalyst for the reduction of $p$-nitrophenol.

It is obvious that the presence of CDs resulted in a two-fold increase in the catalytic rate of the reduction of $p$-nitrophenol when combined with aGNS, which itself is known as a good catalyst for this reaction. ${ }^{9}$ This might be due to the acceleration of the electron transport through the graphitic carbonaceous core of the CDs. ${ }^{15}$ In addition, $p$-nitrophenol is firmly placed within close proximity to the gold surface aided by the hydrophobic carbon chain of the nanocomposite. In fact, the presence of $p$-nitrophenol in the hydrophobic domain was consistent with a slight blue-shift in the UV spectra of the compound in the presence of the aGNS-CD composite in aqueous buffer (Fig. S5, ESI $\dagger$ ). Thus, it is likely that an enhanced capacity for transferring electrons leads to an increase in the catalytic rate of the reduction. However, beyond a certain concentration of CDs in the aGNS-CD nanocomposite, the rate of catalysis remained the same probably due to the lack of penetration space for the reactant molecules to get close to the gold nanosurface.

To this end, we have also observed the ability of this gold-CD nanocomposite to catalyze the reduction of $o$-nitrophenol to $o$-aminophenol. The concentration of $\mathrm{Au}$ and CDs in the nanocomposite were maintained at $50 \mu \mathrm{M}$ and $50 \mu \mathrm{g} \mathrm{mL} \mathrm{m}^{-1}$, respectively. In this case, the progression of this pseudo first order reaction was monitored spectrophotometrically by observing the rate of decrease in the absorbance at $420 \mathrm{~nm}$ (characteristic UV peak of $o$-nitrophenol) (Fig. S6, ESI $\dagger$ ). Here also, the rate of the reduction was increased by 1.6-fold in the presence of CDs. The observed $k_{\text {app }}$ was $0.064 \mathrm{~s}^{-1}$ in only aGNS solution, which increased to $0.104 \mathrm{~s}^{-1}$ in the gold-CD nanocomposite in aqueous buffer (Fig. S6, ESI $\dagger$ ); however, in the absence of the catalyst, almost no reduction occurred.

We also used one water-insoluble substrate ( $m$-dinitrobenzene) to check the efficacy of our designed gold-CD nanocomposite towards its reduction. $m$-Dinitrobenzene was reduced to $m$-phenylenediamine in the presence of $\mathrm{NaBH}_{4}$ and our nanocomposite in aqueous phosphate buffer. ${ }^{14}$ The corresponding change in the UV-vis spectra also demonstrated this fact (Fig. S7, ESI $\dagger$ ). In addition, the gradual blue shift in the UV-vis spectral maxima of $m$-dinitrobenzene ( 244 to $241 \mathrm{~nm}$ ) in the aGNS and gold-CD composite validates the presence of the compound in the hydrophobic domain of the nanocomposite (Fig. S8, ESI $\dagger$ ). In this case also, the pseudo first order rate constant of the reaction was found to be increased by two-fold in the presence of CDS (from $0.14 \mathrm{~s}^{-1}$ to $0.28 \mathrm{~s}^{-1}$ ) (Fig. S9, ESI $\dagger$ ).

In conclusion, we have developed a new class of gold-CD nanocomposites, which show high efficiency in catalyzing the reduction of different aromatic nitro groups. Importantly, to the best of our knowledge, this is the first report of the catalytic properties of carbon dots in conjugation with a gold nanospecies for the reduction of both hydrophilic and hydrophobic nitroarenes. We believe that the presence of CDs increased the rate of electron transfer, as well as secures the localization of the reactant molecules within the catalyst; thus, a two-fold enhancement in catalytic activity in comparison to only aGNS was found in almost every case. This novel catalyst is stable in water and has shown good recyclable efficiency towards catalysis. Most interestingly, the catalysis can also be efficiently performed for water-insoluble compounds, like $m$-dinitrobenzene. Therefore, the presented nanocomposite has potential applications in the design of various industrially important catalysts.

\section{Acknowledgements}

We are thankful to the University Grant Commission (UGC) and the Department of Higher Education, West Bengal for funding this study. We are truly grateful to our Principal, Vice-Principal Maharaj and faculty members of the Chemistry Department.

\section{Notes and references}

1 M. Haruta, Catal. Today, 1997, 36, 153. 
2 (a) G. A. Somorjai, A. M. Contreras, M. Montano and R. M. Rioux, Proc. Natl. Acad. Sci. U. S. A., 2006, 103, 10577; (b) X. Yuan, G. Sun, H. Asakura, T. Tanaka, X. Chen, Y. Yuan, G. Laurenczy, Y. Kou, P. J. Dyson and N. Yan, Chem. - Eur. J., 2013, 19, 1227.

3 (a) M. Boronat and A. Corma, Langmuir, 2010, 26, 16607; (b) A. Jakhmola, R. Bhandari, D. Pacardo and M. R. Knecht, J. Mater. Chem., 2010, 20, 1522; (c) C. Ornelas, J. Ruiz, L. Salmon and D. Astruc, Adv. Synth. Catal., 2008, 350, 837; (d) R. Bhandari and M. R. Knecht, ACS Catal., 2011, 1, 89; (e) V. Polshettiwar, J.-M. Basset and D. Astruc, ChemSusChem, 2012, 5, 6.

4 (a) J. Zeng, Q. Zhang, J. Chenand and Y. Xia, Nano Lett., 2010, 10, 30; (b) J. Lee, J. C. Park, J. U. Bang and H. Song, Chem. Mater., 2008, 20, 5839.

5 (a) J. Zhang, G. Chen, M. Chaker, F. Rosei and D. Ma, Appl. Catal., B, 2013, 107, 132; (b) S. Maiti, M. Ghosh and P. K. Das, Chem. Commun., 2011, 47, 9864.

6 (a) Z. Liu, K. Yang, Y. Liu and S.-T. Lee, J. Mater. Chem., 2012, 22, 24230; (b) J. C. G. E. da Silva and H. M. R. Goncalves, Trends Anal. Chem., 2011, 30, 1327; (c) S. S. Chou, M. De,
J. Luo, V. M. Rotello, J. Huang and V. P. Dravid, J. Am. Chem. Soc., 2012, 134, 16725.

7 K. Das, S. Maiti, M. Ghosh, D. Mandal and P. K. Das, J. Colloid Interface Sci., 2013, 395, 111.

8 (a) S. N. Baker and G. A. Baker, Angew. Chem., Int. Ed., 2010, 49, 6726; (b) J. C. G. E. da Silva and H. M. R. Goncalves, TrAC, Trends Anal. Chem., 2011, 30, 1327.

9 X. Guo, Q. Zhang, Y. Sun, Q. Zhao and J. Yang, ACS Nano, 2012, 6, 1165.

10 N. R. Jana, Small, 2005, 1, 875.

11 A. B. Bourlinos, A. Stassinopoulos, D. Anglos, R. Zboril, V. Georgakilas and E. P. Giannelis, Chem. Mater., 2008, 20, 4539.

12 P. Herves, M. Perez-Lorenzo, L. M. Liz-Marzan, J. Dzubiella, Y. Lub and M. Ballauff, Chem. Soc. Rev., 2012, 41, 5569.

13 (a) R. Jin, Y. Xing, X. Yu, S. Sun, D. Yu, F. Wang, W. Wu and S. Song, Chem. - Asian J., 2012, 7, 2955; (b) S. Rana and K. M. Parida, Catal. Sci. Technol., 2012, 2, 979.

14 A. A. Vernekar, S. Patil, C. Bhat and S. G. Tilve, RSC Adv., 2013, 3, 13243.

15 J. Nanda, A. Biswas, B. Adhikari and A. Banerjee, Angew. Chem., Int. Ed., 2013, 52, 5041. 